\title{
Arranjos configurados pelas vias bilíferas no fígado do avestruz (Struthio camelus)
}

\section{Arrangement configured by bile duct in the liver of the ostrich (Struthio camelus)}

\author{
Ana Julia Silva e ALVES ${ }^{1}$; Wilson Machado de SOUZA ${ }^{1}$; Nair Trevizan Machado de SOUZA ${ }^{1}$; Adão \\ Ângelo CUSTÓDIO'
}

${ }^{1}$ Faculdade de Medicina Veterinária da Universidade Estadual Paulista “Julio de Mesquita Filho”, Araçatuba-SP, Brasil

\begin{abstract}
Resumo
A ave avestruz (Struchio camelus) é originária da África, pertencendo a família das Ratitas. Seu valor zootécnico vem crescendo especialmente na região noroeste do Estado de São Paulo. Para a realização deste trabalho, utilizamos 20 peças de avestruz (Struthio camelus), machos e fêmeas, jovens e adultos, oriundos de criatórios da região noroeste do Estado de São Paulo. Após a abertura do trato gastrintestinal, ao longo da sua margem livre, escoamos as vias bilíferas mediante suaves massagens, canalizamos o ducto excretor, utilizando para tanto uma sonda flexível de polietileno de calibre compatível com a mencionada estrutura e, em seguida, injetamos por esta via Neoprene-látex “450” corado. Estas glândulas foram, a seguir, distendidas em base rígida, fixadas em solução de formol a $10 \%$, esquematizadas e fotografadas. O ramo principal direito resulta do ramo lateral direito, ramo lobo quadrado e do ramo processo caudado em sete fígados. Em 13 peças, o ramo lateral direito é formado pelos ramoslateral direito e do processo caudado.O ramo esquerdo recebe os componentes oriundos do lobo lateral esquerdo e ramo do lobo medial esquerdo em todas as sete peças. Para ele, também aflui componente oriundo do lobo quadrado em 13 preparações e do processo caudado em sete fígados. Conclui-se que ofígado no avestruz não apresenta vesícula biliar, fazendo com que o ducto colédoco seja a única via de excreção da bile, resultando sempre da convergência do ducto hepático direito com o ducto hepático esquerdo.
\end{abstract}

Palavras-chave: Sistema biliar. Avestruz. Fígado. Struthio camelus. Glândula.

\begin{abstract}
The aim of this study was evaluate the influence of physical exercise (marcha gait) on serum values of CK and AST The Ostrich (Struthio camelus) is originally from Africa and belongs to the ratite's family. Since its zootechnic value has been increasing, especially in the northwest region of the state of São Paulo, we studied the biliary system in the liver of this animal. To carry through it were used 20 ostrich livers, males and females, young and adults from place to production ostrich in the northwest region of the State of São Paulo. After the opening of the gastrointestinal tract and disposal of the bile through gentle massage, we cannulated the hepatic excretory duct with a flexible probe, compatible in size with the duct, and injected colored Neoprene latex " 450 " in the biliary system. Following this procedure, the livers were fixed in aqueous solution of formaldehyde to $10 \%$ dissected, schematized and photographed. The right main branch is derived from the right lateral, square lobe and the caudate process branches in 7 livers. In 13 livers, the right side branch is forned by the right side and caudate process branches. The left branch receives the components from the left lateral lobe and branch of the medial left lobe in 7 livers. It also receives component from the square lobe and from the caudate process in 13 and 7 livers, respectively.In conclusion, the ostrich liver has no gallbladder, causing the duct is the only route of excretion of bile, always resulting from the convergence of the right hepatic duct with the left hepatic duct.
\end{abstract}

Keywords: Biliary system. Ostrich. Liver. Struthio camelus. Gland.

\section{Introdução}

É provável que as aves tenham surgido há 180 milhões de anos no período jurássico, com uma vantagem sobre os demais indivíduos, o poder de voar, que lhe proporcionou condições de alcançar quase todos os nichos ecológicos ${ }^{1}$.
De modo geral, é aceito que as aves se diferenciaram a partir dos répteis, bem mais tarde que os mamíferos,

Correspondência para:

Ana Julia Silva e Alves

rabin.alves@gmail.com

Recebido: 14/06/11

Aprovado: 20/12/12 
sendo, portanto, mais parecidas anatomicamente com os primeiros. O voo impõe sobre a estruturação destes animais uma quase simetria na topografia dos órgãos, embora nem todos os indivíduos deste grupo voem como é o caso dos pinguins e ratitas, onde se incluem a ema, avestruz, emus e casuar, entre outros. O termo ratita refere-se ao osso externo com carena bastante reduzida ${ }^{1,2}$.

Particularizando o avestruz (Struthio camelus), este é originário da África e pertencendo a família das Ratitas - aves não voadoras. Por diversas qualidades, vem se tornando uma alternativa aos avicultores, pois a sua carne está sendo comparada, embora seja uma ave, com a carne vermelha bovina, cujo gosto e maciez são semelhantes ao do filet mignon, porém com níveis de calorias, gordura e colesterol muito mais baixos ${ }^{3}$.

Esta ave apresenta um valorzootécnico relacionado não apenas à produção de carne, mas também ao aproveitamento da pele (cútis) e de suas penas. Outros mercados podem ser explorados, como os ovos inférteis, aproveitando a casca como artesanato e o conteúdo para consumo humano ou para a indústria de alimento, pois comparado aos ovos de galinha possui um menor teor de gordura e maior relação de aminoácidos essenciais (6585mg em 100 gramas no ovo de avestruz e $5837 \mathrm{mg}$ em 100 gramas no ovo de galinha) ${ }^{3}$.

A Estruticultura é considerada como atividade agropecuária que demanda pouco em instalações e o seu sucesso de está voltado nos investimentos na qualidade de plantel, manejo alimentar e sanitário dos animais ${ }^{3}$.

Evidentemente, atividades zootécnicas ainda em implantação no Brasil exigem conhecimentos pormenorizados do animal a ser explorado. Particularizando o avestruz, existe em nosso meio um paralelo muito grande em relação à ema, mas pouco se conhece sobre a morfofisiologia deste animal, embora estes dados sejam fundamentais quando se pensa em explorar racionalmente qualquer espécie animal.

Assim, pela escassez de dados sobre as vias bilíferas, objetivou-se empreender um estudo sobre a anatomia destas estruturas no fígado deste animal, objetivando compará-las com os estudos já existentes em outras aves.

\section{Material e Método}

Para a realização deste trabalho, foram utilizadas 20 peças de avestruz (Struthio camelus), machos e fêmeas, jovens e adultos, oriundos do criatório da empresa Aravestruz do Estado de São Paulo, mais especificamente da cidade de Araçatuba, durante todo o ano de 2005.

Cada uma destas preparações compreende o fígado e porção do intestino delgado, correspondente à desembocadura do ducto excretor da glândula.

Após a abertura do referido trato gastrointestinal, ao longo da sua margem livre, foram escoadas as vias bilíferas mediante suaves massagens, canalizamos $o$ ducto biliar, utilizando para tanto uma sonda flexível de polietileno de calibre compatível para injeção de Neoprene-látex “450” corado.

Estas glândulas foram, a seguir, distendidas em base rígida, fixadas em solução de formol a $10 \%$, por um período mínimo de 24 horas para em seguida serem dissecados pela face visceral.

Todos os fígados analisados foram esquematizados e alguns deles, aleatoriamente, foram fotografados (Figuras 1, 2, 3).

Os resultados obtidos nesta pesquisa foram expressos conforme recomendações da Nomina Anatômica Avium ${ }^{4}$, verificando as alusões aos trabalhos de $\mathrm{Pa}$ checo, Prada e Borelli ${ }^{5}$ e Jablan-Pantic e Antonievic ${ }^{6}$.

\section{Resultados}

\section{Morfologia externa do órgão}

A morfologia externa do fígado de avestruz permite identificar uma lobação característica para a espécie, constituída pelo lobo lateral direito, processo caudado do lobo caudado, lobo lateral esquerdo, lobo medial esquerdo e lobo quadrado, além de mostrar uma profunda fissura mediana que constitui estrutura de reparo para se estabelecer os componentes direito e esquerdo 


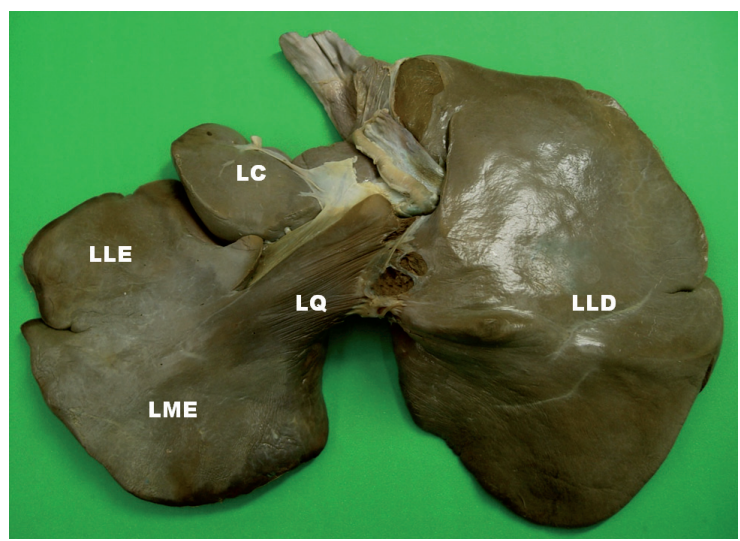

Figura 1 - Fotografia do fígado de Avestruz, fixado em solução de formol a 10 \%, mostrando a morfologia externa do órgão, se observado, pela face visceral, a lobação desta glândula, designados como: LLE- lobo lateral esquerdo; LME- lobo medial esquerdo; LQ- lobo quadrado; LLD- lobo lateral direito; LC- lobo caudado - Araçatuba-2005

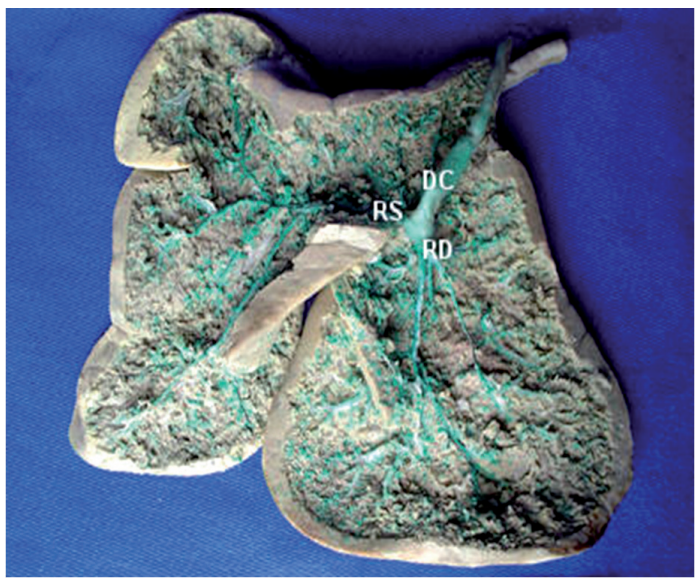

Figura 2 - Fotografia do fígado de Avestruz, cujas vias bilíferas foram injetadas com Látex corado em verde, seguindo-se de fixação do parênquima hepático em solução de formol a 10\% e a seguir dissecadas pela face visceral onde identificamos: DC- ducto colédoco; resultando da confluência do RS- ramo principal esquerdo e RD- ramo principal direito - Araçatuba-2005



Figura 3 - Esquema representativo das vias bilíferas no fígado da Avestruz visto pela face visceral, onde se observa a confluência do ramo principal esquerdo (RS), responsável pela drenagem biliar de território hepático de maior extensão com o ramo principal direito (RD) para constituir o ducto colédoco (DC) - Araçatuba- 2005 
desta glândula (Figura 1). Ofígado apresenta uma face parietal convexa, situada craniaventralmente,e uma visceral, côncava de situação caudoventral.

\section{Ducto Colédoco}

O fígado de avestruz não apresenta vesícula biliar (Figura 1) e em todas as peças analisadas, o ducto colédoco emerge a partir da confluência do ramo principal direito e do ramo principal esquerdo.

\section{1- Ramo Principal Direito}

O ramo principal direito resulta do ramo lateral direito, ramo lobo quadrado e do ramo processo caudado em sete fígados. Em 13 peças, o ramo lateral direito é formado pelos ramoslateral direito e do processo caudado (Tabela 1 e Figuras 2 e 3).

\section{2- Ramo Principal Esquerdo}

O ramo esquerdo recebe os componentes oriundos do lobo lateral esquerdo e ramo do lobo medial esquerdo em todas as sete peças. Para ele, também aflui componente oriundo do lobo quadrado em 13 preparações e do processo caudado em sete fígados (Tabela 2, Figuras 2 e 3).

\section{Discussão}

Os resultados encontrados em relação às vias bilíferas do fígado de avestruz (Struthio camelus) mostram o ducto colédoco como único coletor a afluir para o duodeno, informe compatível com a observação da Nomina Anatômica Avium ${ }^{4}$ (1993). Evidentemente, este fato é diretamente relacionado com a ausência da vesícula biliar na espécie, não permitindo assim um confronto direto com as alusões dos livros clássicos 2 , 5,6,7, 8, 9, 10, 11, 12, $13,14,15,16,17,18,19,20$, pois todos estes autores registram nas aves por eles estudadas, duas vias de drenagem biliar para o duodeno designadas de maneira diversas pelo primeiro grupo e de ducto cisticoentérico e ducto hepatoentérico, pelos do segundo.

De fato, a ausência da vesícula biliar é um dado relevante no estudo da morfologia do fígado nas es-

Tabela 1 - Composição do duto hepático direito no fígado de avestruz (Struthio camelus) Araçatuba-2005

\begin{tabular}{|c|c|}
\hline Obs. & Procedência dos ramos formadores do ducto hepático direito \\
\hline 1 & Lobo lateral direito, lobo quadrado, processo caudatum \\
\hline 2 & Lobo lateral direito, lobo quadrado, processo caudatum \\
\hline 3 & Processo Caudado, Lobo Direito \\
\hline 4 & Lobo lateral direito, lobo quadrado, processo caudatum \\
\hline 5 & Lobo lateral direito, lobo quadrado, processo caudatum \\
\hline 6 & Lobo lateral direito, lobo quadrado, processo caudatum \\
\hline 7 & Processo Caudado, Lobo Direito \\
\hline 8 & Processo Caudado, Lobo Direito \\
\hline 9 & Lobo lateral direito, lobo quadrado, processo caudatum \\
\hline 10 & Processo Caudado, Lobo Direito \\
\hline 11 & Processo Caudado, Lobo Direito \\
\hline 12 & Processo Caudado, Lobo Direito \\
\hline 13 & Processo Caudado, Lobo Direito \\
\hline 14 & Processo Caudado, Lobo Direito \\
\hline 15 & Processo Caudado, Lobo Direito \\
\hline 16 & Processo Caudado, Lobo Direito \\
\hline 17 & Processo Caudado, Lobo Direito \\
\hline 18 & Lobo lateral direito, lobo quadrado, processo caudatum \\
\hline 19 & Processo Caudado, Lobo Direito \\
\hline 20 & Processo Caudado, Lobo Direito \\
\hline
\end{tabular}


Tabela 2 - Composição do duto hepático esquerdo no fígado de avestruz (Struthio camelus) Araçatuba-2005

\begin{tabular}{cc}
\hline Obs & Ramo Lateral Esquerdo \\
\hline $\mathbf{1}$ & Lobo lateral esquerdo, lobo medial esquerdo \\
$\mathbf{2}$ & Lobo lateral esquerdo, lobo medial esquerdo \\
$\mathbf{4}$ & Lobo medial esquerdo, lateral esquerdo, lobo quadrado \\
$\mathbf{5}$ & Lobo medial esquerdo, lateral esquerdo, lobo quadrado \\
$\mathbf{6}$ & Lobo medial esquerdo, lateral esquerdo, lobo quadrado \\
$\mathbf{7}$ & Lobo medial esquerdo, lateral esquerdo, lobo quadrado \\
$\mathbf{8}$ & Lobo medial esquerdo, lateral esquerdo, lobo quadrado \\
$\mathbf{9}$ & Lobo medial esquerdo, lateral esquerdo, lobo quadrado \\
$\mathbf{1 0}$ & Lobo lateral esquerdo, lobo medial esquerdo \\
$\mathbf{1 1}$ & Lobo medial esquerdo, lateral esquerdo, lobo quadrado \\
$\mathbf{1 2}$ & Lobo lateral esquerdo, lobo medial esquerdo \\
$\mathbf{1 3}$ & Lobo lateral esquerdo, lobo medial esquerdo \\
$\mathbf{1 4}$ & Lobo medial esquerdo, lateral esquerdo, lobo quadrado \\
$\mathbf{1 5}$ & Lobo medial esquerdo, lateral esquerdo, lobo quadrado \\
$\mathbf{1 6}$ & Lobo lateral esquerdo, lobo medial esquerdo \\
$\mathbf{1 7}$ & Lobo medial esquerdo, lateral esquerdo, lobo quadrado \\
$\mathbf{1 8}$ & Lobo medial esquerdo, lateral esquerdo, lobo quadrado \\
$\mathbf{1 9}$ & Lobo medial esquerdo, lateral esquerdo, lobo quadrado \\
$\mathbf{2 0}$ & Lobo medial esquerdo, lateral esquerdo, lobo quadrado \\
\hline
\end{tabular}

pécies, pois conforme esclarece Romer e Parsons ${ }^{1}$, ao surgirem a partir dos répteis no período jurássico, as aves tiveram acesso aos diferentes nichos ecológicos pela capacidade de voar, não adquirida ou perdida pelos emus, emas e avestruzes que, entretanto, ganharam a capacidade de se deslocar em grande velocidade, como, aliás, ocorre entre alguns mamíferos como, equídeos, cervídeos e certos carnívoros, que também são desprovidos da referida estrutura. Talvez possa existir aí um paralelo entre estes dois grupos, vale dizer, a velocidade como um meio de sobrevivência no ambiente e a ausência da vesícula biliar.

É fácil verificar a não existência da vesícula biliar na espécie e,consequentemente, a não caracterização dos ductos hepatoentérico e cisticoentérico, tornam a descrição das vias excretoras do fígado mais simples, pois o ducto colédoco resulta sempre (100\%) da convergência do ramo principal direito e do ramo principal esquerdo. O primeiro destes é constituído pela confluência apenas do ramo processo caudado e do lobo direito em $65 \%$ das preparações, enquanto nas demais 35\% a estes coletores soma-se o ramo lobo quadrado. O ducto hepático esquerdo, por sua vez, procede da junção dos ramos lateral esquerdo, medial esquerdo e quadrado na maioria das vezes (65\%), já nas demais glândulas $(35 \%)$, o referido coletor resulta da confluência dos ramos lateral esquerdo e medial esquerdo. Estes dados, se tomados genericamente, não se afastam daqueles assinalados $4,6,16,17,18,19,20$.

À situação topográfica do fígado, são fundamentais os informes de alguns autores ${ }^{21,22,23}$, os dois primeiros referentes aos gallus e o último ao avestruz. Estes autores revelam que o fígado nas aves mostra face parietal convexa, situada cranioventralmente, e uma visceral, côncava de situação caudoventral. Estas informações não destoam das nossas observações relativas ao avestruz. 
A importância do fígado como glândula anexa ao tubo digestório e participante fundamental em outras funções do organismo dos cordados, já pode ser observada quando Storer et al. ${ }^{24}$ assinala a presença do órgão na forma rudimentar ao longo deste tubo no anfioxo. Neste sentido, também chama-nos atenção as alusões de Nickel, Schummer e Seiferle ${ }^{22}$ quando informam que o fígado apresenta tamanho, consistência e coloração dependente da raça, idade e estado nutricional do indivíduo.

\section{Conclusões}

Do estudo que efetuamos sobre as vias bilíferas em 20 fígados de avestruz (Struthio camelus), julgamos poder concluir que:

O fígado no avestruz não apresenta vesícula biliar,

\section{Referências}

1. ROMER, A. S.; PARSONS, T. S. Anatomia comparada dos vertebrados. São Paulo: Atheneu, 1985.

2. GETTY, R. Sisson and Grossman is the anatomy of de domestic animals. 5. ed. Philadelphia: Saunders, 1975. v. 2, p. $1878-1879$.

3. CARRER, C. C.; KORNFELD, M. E. A criação de avestruzes. Pirassununga: C. C. Carrer, 1999. p. 27-40.

4. BAUMEL, J.; KING, A. S.; LUCAS, A. M.; BREAZILE, J. E.; EVANS, H. E. Nomina anatomia avium. London: Academic Press, 1979.

5. PACHECO, Y. G.; PRADA, I. L. S.; BORELLI, V. Contribuicão ao estudo das vias bilíferas intra e extra-hepáticas de Gallus gallus domesticus. Revista da Faculdade de Medicina Veterinária e Zootecnia da Universidade de São Paulo, v. 22, n. 1, p. 15-30, 1985.

6. JABLAN-PANTIC, D.; ANTONIEVIC, N. Intrahepatic gallenwage des hansgeflugels. Acta Anatonica, v. 10, p. 91102,1960 .

7. LESBRE, F. X. Précis $\mathbf{d}$ anatomie comparee des animaux domestiques. Paris: J.B. Bailliére, 1922. v. 1, p. 679.

8. CARADONNA, G. B. Apparechio gastro-pulmonare. In: ZIMMERL, U. Trattato anatonia veterinaria. Milano: Francesco Vallardi, 1930. v. 2, p. 611-612.

9. ELLENBERGER, W.; BAUM, H. Handbuch der vergleichenden der anatomie der haustiere. Berlim: Julius Springer, 1932. v. 8, p. 1030.

10.PORTMAN, A. Le tube digestif. In: GRASSÉ, P. P. Traité de zoollogie: anatomie, systematique, biollogie. Paris: Masson, 1950. v. 15 , p. 283.

11.SISSON, S.; GROSSMAN, J. D. The anatomy of the domestic animals. 4. ed. Philadelphia: Saunders, 1945. p. 916.

12.GONZALEZ, Y.; GARCIA, J.; GONZALEZ ALVAREZ, R. Anatomia comparada de los animales domesticos. 7. ed. Madrid: Canales, 1961. p. 499-451. não permitindo assim caracterizar os ductos hepatoentérico e cisticoentérico, situação comum em diversos outros gêneros de aves ${ }^{4}$

A ausência da vesícula biliar faz com que o ducto colédoco seja a única via de excreção da bile, resultando sempre da convergência do ducto hepático direito com o ducto hepático esquerdo.

Ao primeiro destes afluem os ramos lobo direito e lobo caudado (65\%), e, além destes, o ramo lobo quadrado (35\%), enquanto ao segundo convergem os ramos medial esquerdo, lateral esquerdo e quadrado (65\%)e apenas os dois primeiros $35 \%$.

E nestas aves o fígado mostra uma face parietal convexa, situada cranioventralmente, e uma visceral, côncava de situação caudoventral.

13. EDE, D. A. Anatomia de las aves. Zaragoza: Acribia, 1965. p. 80.

14. HOFFMAN, G.; VOLKER, H. Anatomia y fisiologia de las aves domésticas. Zaragoza: Acribia, 1968. p. 74.

15.SCHWARZE, E.; SCHRODER, L. Compendio de anatomia veterinária: anatomia de las aves. Zaragoza: Acribia, 1966. v. 5 , p. $89-91$.

16. MIYAKI, T. The hepatic lobule and its relation to the distribution of blood vessels and bile ducts in the fowl. Japanese Journal Veterinary Science, v. 35, p. 403-410, 1973.

17.PINTO E SILVA, P.; JOAQUIM, C. F.; MELLO DIAS, S.; CAMPOS, V. J. M. Contribuição ao estudo das vias biliferas em Gallus gallus. Científica, v. 6, n. 3, p. 431-436, 1978.

18. GUPTA, S. C.; GUPTA, C. D.; GUPTA, S. B. Intrahepatic patterns of the biliary ducts in chick liver (Gallus gallus domesticus). Anatomischer Anzeiger, v. 151, n. 1, p. 64-69, 1982.

19. PACHECO, Y. G.; PRADA, I. L. S. Contribuição ao estudo das vias bilíferas intra e extra-hepáticas de Peru (Mêlleagris gallopavo). In: CONGRESSO BRASILEIRO DE ANATOMIA, 14., 1985, Vitótia. Anais... Vitória: SBA, 1985. p. 79.

20.CANELLAS, L. F. L.; SOUZA, W. M.; SOUZA, N. T. M.; CARVALHAL, R.; MIGLINO, M. A. Estudo anatômico das vias bilíferas na galinha Angola. Ars Veterinária, v. 8, n. 2, p. 83-89, 1992.

21. MCLELLAND, J.; KING, A. S. The gross anatomy of the peritoneal coelomic cavities of Gallus domesticus. Anatomischer Anzeiger, v. 127, n. 5, p. 480-490, 1970.

22. NICKEL, R.; SCHUMMER, A.; SEIFERLE, E. Anatomy of the domestic birds. Berlin: Hamburg: Parey, 1977.

23. BEZUIDENHOUT, A. J. The topography of the thoracoabdominal viscera in the ostrich(Struthio camelus). Onderstepoort Journal Veterinary Research, v. 53, n. 2, p. 111-117, 1986.

24.STORER, T. I.; USINGER, R. L.;STEBBINS, R. C.; NYBAKKEN, J. W. Zoologia geral. 6. ed. São Paulo: Companhia Editora Nacional, 2000. 